\title{
On the Representation of Neutrosophic Matrices by Neutrosophic Linear Transformations
}

\author{
Mohammad Abobala \\ Faculty of Science, Department of Mathematics, Tishreen University, Latakia, Syria \\ Correspondence should be addressed to Mohammad Abobala; mohammadabobala777@gmail.com
}

Received 8 January 2021; Revised 25 January 2021; Accepted 9 February 2021; Published 25 February 2021

Academic Editor: Parimala Mani

Copyright (c) 2021 Mohammad Abobala. This is an open access article distributed under the Creative Commons Attribution License, which permits unrestricted use, distribution, and reproduction in any medium, provided the original work is properly cited.

The objective of this paper is to study the representation of neutrosophic matrices defined over a neutrosophic field by neutrosophic linear transformations between neutrosophic vector spaces, where it proves that every neutrosophic matrix can be represented uniquely by a neutrosophic linear transformation. Also, this work proves that every neutrosophic linear transformation must be an $\mathrm{AH}$-linear transformation; i.e., it can be represented by classical linear transformations.

\section{Introduction}

Neutrosophy is a new branch of philosophy founded by Smarandache $[1,2]$ to deal with uncertainty in real-life problems.

Neutrosophic concepts found their way in many other fields, such as classification [3, 4], number theory [5, 6], algebraic equations [7, 8], Boolean algebra [9] and optimization [10].

Neutrosophic algebra began with Smarandache and Kandasamy in [11], where they defined neutrosophic rings and fields for the first time. Lately, neutrosophic fields [12] were used in the study of neutrosophic vector spaces [13-16].

Neutrosophic matrices were defined to deal with indeterminacy problems, and many applications and theorems can be found in [17-19].

If $\mathrm{V}$ is a vector space over the field $\mathrm{F}$, then $V(I)=\{x+y I ; x, y \in V\}$ is the corresponding strong neutrosophic vector space over the neutrosophic field $F(I)$.

In [4, 20-24], Abobala et al. proposed the concept of $\mathrm{AH}$ substructures in groups, rings, spaces, and modules as a neutrosophic structures with two classical parts; for example, in the strong neutrosophic vector space $V(I)$, an $\mathrm{AH}$ subspace is the set $W(I)=T+S I$, where $T$ and $S$ are two classical subspaces of $V$. In a similar way, an $\mathrm{AH}$ linear transformation is a function $f$ between two neutrosophic vector spaces $V(I)$ and $W(I)$ with two classical parts $f=g+h I$, where $g$ and $h$ are classical linear transformations between $\mathrm{V}$ and $\mathrm{W}$.

It is known that classical matrices can be represented by linear transformations; from this point of view, we will study this problem in single valued neutrosophic systems.

In this work, we study neutrosophic matrices as linear neutrosophic functions. In particular, we prove that every linear transformation between two neutrosophic vector spaces must have an $\mathrm{AH}$ structure.

\section{Preliminaries}

Definition 1 (see $[16])$. Let $(\mathrm{V},+,$.$) be a vector space over the$ field $\mathrm{K}$, then $(V(I),+,$.$) is called a weak neutrosophic vector$ space over the field $\mathrm{K}$, and it is called a strong neutrosophic vector space if it is a vector space over the neutrosophic field $K(I)$.

A neutrosophic field $K(I)$ is a triple $(K(I),+,$.$) , where \mathrm{K}$ is a classical field. A neutrosophic field is not a field by classical meaning, but it is a ring.

Elements of $V(I)$ have the following form: $\mathbf{x}+\mathbf{y I} ; \mathbf{x}$, $\mathbf{y} \in \mathbf{V}$; i.e., $V(I)$ can be written as $\mathbf{V}(\mathbf{I})=\mathbf{V}+\mathbf{V I}$.

Definition 2 (see [16]). Let $V(I)$ be a strong neutrosophic vector space over the neutrosophic field $K(I)$ and $W(I)$ be a 
nonempty set of $V(I)$, then $W(I)$ is called a strong neutrosophic subspace if $W(I)$ itself is a strong neutrosophic vector space.

Definition 3 (see [16]). Let $v_{1}, v_{2} . v_{s} \in V(I)$, and $x \in V(I)$ we say that $x$ is a linear combination of $\left\{v_{i} ; i=1, \ldots, s\right\}$ if

$$
x=a_{1} v_{1}+\ldots+a_{s} v_{s} \operatorname{such} a_{i} \in K(I) .
$$

The set $\left\{v_{i} ; i=1, . ., s\right\}$ is called linearly independent if $a_{1} v_{1}+\cdots+a_{s} v_{s}=0$ implies $a_{i}=0$ for all $i$.

Definition 4 (see [18]). Let $M_{m \times n}=\left\{\left(a_{i j}\right): a_{i j} \in K(I)\right\}$ where $K(I)$ is a neutrosophic field. We call it the neutrosophic matrix.

\section{Main Discussion}

Theorem 1. Let $V, W$ be two vector spaces over the field $F$ with $\operatorname{dim}(V)=n, \operatorname{dim}(W)=m$ and $V(I), W(I)$ be the corresponding neutrosophic vector spaces over the corresponding neutrosophic field $F(I)$. Let $g, h: V \longrightarrow W$ be two linear transformations, then there exists a neutrosophic linear transformation $f=g+h I: V(I) \longrightarrow W(I)$, where $f$ is defined as follows:

$$
f(x+y I)=g(x)+[(g+h)(x+y)-g(x)] I .
$$

Proof. We define $f=g+h I: V(I) \longrightarrow W(I)$, where

$$
f(x+y I)=g(x)+[(g+h)(x+y)-g(x)] I,
$$

in which $f$ is a linear transformation, that is, because for every $m=x+y I, n=z+t I \in V(I)$, we have

$$
\begin{aligned}
f(m+n)= & f([x+z]+I[y+t])=g(x+z) \\
& +I[(g+h)(x+y+z+t)-g(x+z)] \\
= & (g(x)+[(g+h)(x+y)-g(x)] I) \\
& +(g(z)+[(g+h)(z+t)-g(z)] I) \\
= & f(m)+f(n) .
\end{aligned}
$$

On the contrary, consider an arbitrary neutrosophic number $a+b I \in F(I)$, then

$$
\begin{aligned}
f([a+b I] m) & =f([a+b I][x+y I])=f(a x+I[a y+b x+b y])=f(a x+I[(a+b)(x+y)-a x]) \\
& =g(a x)+I[(g+h)[(a+b)(x+y)]-g(a x)] \\
& =a g(x)+I[(a+b)(g+h)[x+y]-a g(x)] \\
& =(a+b I)(g(x)+I[(g+h)(x+y)-g(x)]=(a+b I) f(m) .
\end{aligned}
$$

Thus, $f$ is a neutrosophic linear transformation.

Definition 5. The neutrosophic linear transformation $f$ defined in Theorem 1 is called a full AH-linear transformation.

Definition 6. Let $f=g+h I: V(I) \longrightarrow W(I)$ be a full AHlinear transformation and $M=A+B I$ be an $n \times m$ neutrosophic matrix over $F(I)$, and we call $M$ the neutrosophic matrix of $f$ if and only if $f(x+y I)=M(x+y I)$ for every $x+y I \in V(I)$.
Theorem 2. Let $f=g+h I: V(I) \longrightarrow W(I)$ be any full AH-linear transformation, then $M=A+B I$ is the corresponding neutrosophic matrix if and only if $A$ is the matrix of $g$ and $B$ is the matrix of $h$.

Proof. We assume that A is the matrix of $g$ and B is the matrix of $h$; hence, $A x=g(x), \mathrm{By}=h(y),(A+B)$ $(x+y)=(g+h)(x+y)$. We have

$$
\begin{aligned}
M .(x+y I) & =(A+B I)(x+y I)=(A x+I[A y+B x+B y])=(A x+I[(A+B)(x+y)-A x]) \\
& =g(x)+I[(g+h)(x+y)-g(x)]=f(x+y I) .
\end{aligned}
$$

Thus, $M$ is the neutrosophic matrix of $f$.

Conversely, suppose that $M$ is the neutrosophic matrix of $f$, and we shall prove that $\mathrm{A}$ is the matrix of $g$ and $\mathrm{B}$ is the matrix of $h$.

According to the assumption, we have $M(x+y I)=$ $f(x+y I)$; hence,

$$
\begin{aligned}
& (A x+I[(A+B)(x+y)-A x]) \\
& \quad=g(x)+I[(g+h)(x+y)-g(x)] .
\end{aligned}
$$

This implies that $A x=g(x),(A+B)(x+y)=(g+h)$ $(x+y)$ so that $B(x+y)=h(x+y)$. By considering the arbitrariness of $x$ and $y$, we get that A is the matrix of $g$ and $\mathrm{B}$ is the matrix of $h$. 
Example 1

(a) Let $V(I)=R^{2}(I)=\{(a, b)+(c, d) I=(a+c I, b+$ $d I) ; a, b, c, d \in R\}$, consider the following neu- corresponding neutrosophic linear transformation is defined as follows:

trosophic matrix $M=\left(\begin{array}{cc}1+I & I \\ -I & 2-I\end{array}\right) . \quad$ The

$$
\begin{aligned}
f(x+y I) & =M \cdot\left(\begin{array}{c}
a+c I \\
b+d I
\end{array}\right)=(a+I[c+a+c+b+d],-a I-c I+2 b+2 d I-b I-d I) \\
& =(a+I[a+2 c+b+d], 2 b+I[-a-c-b+d])=(a, 2 b)+I(a+2 c+b+d,-a-c-b+d) .
\end{aligned}
$$

(b) $f=g+h I ; g(x, y)=(x, 2 y), h(x, y)=(x+y,-x-y)$, where $g, h: V \longrightarrow V$.

Theorem 3. Let $V, W$ be two vector spaces over the field $F$, with $\operatorname{dim}(V)=n, \operatorname{dim}(W)=m$, and let $M=A+B I$ be any $n \times m$ neutrosophic matrix over $F(I)$. Then, $M$ can be represented by a unique full AH-linear transformation $f=g+h I$, where $A$ is the matrix of $g$ and $B$ is the matrix of $h$.

Proof. According to Theorem 2, the neutrosophic matrix $M=$ $A+B I$ can be represented by a neutrosophic full AH-linear transformation $f=g+h I$, where $\mathrm{A}$ is the matrix of $g$ and $\mathrm{B}$ is the matrix of $h$. For the uniqueness condition, we suppose that $F=G+H I$ is another linear AH-transformation with the property.

$$
\begin{aligned}
& M(x+y I)=F(x+y I) . \text { We have } \\
& M .(x+y I)=F(x+y I)=f(x+y I), \\
& \text { for all } x+y I \in V(I) .
\end{aligned}
$$

Thus, $F=f$ and $f$ is unique.

The following theorem shows an algorithm to find a basis for the neutrosophic vector space $V(I)$ from any basis of the corresponding classical vector space $V$.

Theorem 4. Let $V(I)$ be any neutrosophic vector space over the neutrosophic field $F(I)$ and $V$ be its corresponding classical vector space over the field F. Let $S=\left\{v_{1}, v_{2}, \ldots, v_{n}\right\}$ be a basis of $V$ over $F$, then $L=\left\{l_{i j}=v_{i}+\left(v_{j}-v_{i}\right) I ; 1 \leq i, j \leq n\right\}$ is a basis of $V(I)$ over $F(I)$.

Proof. First of all, we must prove that $L$ generates $V(I)$ over $F(I)$. Let $x+y I$ be any element of $V(I)$, where $x, y \in V$, and we have

$$
\begin{aligned}
x & =\sum_{i=1}^{n} a_{i} v_{i}, x+y=\sum_{j=1}^{n} b_{j} v_{j}, \text { We put } r_{i j} \\
& =a_{i}+\left(b_{j}-a_{i}\right) I \in F(I) .
\end{aligned}
$$

Now, we compute $\sum_{i, j=1}^{n} r_{i j} l_{i j}$.

$$
\begin{aligned}
\sum_{i, j=1}^{n} r_{i j} l_{i j} & =\sum_{i, j=1}^{n}\left(a_{i}+\left(b_{j}-a_{i}\right) I\right)\left(v_{i}+\left(v_{j}-v_{i}\right) I\right) \\
& =\sum_{i, j=1}^{n} a_{i} v_{i}+I\left[b_{j} v_{j}-a_{i} v_{i}\right] \\
& =\sum_{i=1}^{n} a_{i} v_{i}+I\left[\sum_{j=1}^{n} b_{j} v_{j}-\sum_{i=1}^{n} a_{i} v_{i}\right] \\
& =x+I[(x+y)-x]=x+y I .
\end{aligned}
$$

Thus, $L$ generates $V(I)$ over $F(I)$.

Now, we prove that $L$ is linearly independent. For this purpose, we assume that $\sum_{i, j=1}^{n}\left(a_{i}+b_{j} I\right) l_{i j}=0$; thus, we get

$$
\begin{aligned}
\sum_{i, j=1}^{n}\left(a_{i} v_{i}+I\left[\left(a_{i}+b_{j}\right) v_{j}-a_{i} v_{i}\right)=0, \text { hence, } \sum_{i=1}^{n} a_{i} v_{i}\right. \\
=\sum_{i, j}^{n}\left(a_{i}+b_{j}\right) v_{j}=0 \text {, thus, } a_{i}=a_{i}+b_{j}=0 \text {, so that, } b_{j}=0 .
\end{aligned}
$$

This implies that $L$ is linearly independent, and then it is a basis.

Example 2. It is well known that $\{x=(1,0), y=(0,1)\}$ is a basis of $\mathrm{V}=R^{2}$. The corresponding basis of $V(I)=R^{2}(I)$ is

$$
\begin{aligned}
& \{x, y, x+(y-x) I, y+(x-y) I\} \\
& \quad=\{(1,0),(0,1),(1,0)+(-1,1) I,(0,1)+(1,-1) I\} .
\end{aligned}
$$

The following theorem shows that every linear transformation between $V(I)$ and $W(I)$ must be a full AH-linear transformation.

Theorem 5. Let $V, W$ be two vector spaces over the field $F$, with $\operatorname{dim}(V)=n, \operatorname{dim}(W)=m$ and let $V(I), W(I)$ be the corresponding neutrosophic vector spaces over $F(I)$. Let $f: V(I) \longrightarrow W(I)$ be any linear transformation, then $f$ is a full AH-linear transformation.

Proof. Let $f: V(I) \longrightarrow W(I)$ be any linear transformation, and we must prove that there exists two classical linear transformations $g, q: V \longrightarrow W$, where $f=g+q I$. 
Suppose that $S=\left\{v_{1}, v_{2}, \ldots, v_{n}\right\}$ is a basis of $\mathrm{V}$, then $L=$ $\left\{l_{i j}=v_{i}+\left(v_{j}-v_{i}\right) I ; 1 \leq i, j \leq n\right\}$ is a basis of $V(I)$. It is known that $f(L)=\left\{f\left(v_{i}+\left(v_{j}-v_{i}\right) I\right)=w_{i}+\left(w_{j}-w_{i}\right)\right.$ $\left.I ; w_{i}, w_{j} \in W\right\}$ is a basis of $W(I)$ and that is because the direct image of a basis by any linear transformation is a gain a basis.

Define $g: V \longrightarrow W ; g\left(v_{i}\right)=w_{i}, h: V \longrightarrow W ; h\left(v_{j}\right)=w_{j}$. It is clear that $f\left(v_{i}+\left(v_{j}-v_{i}\right) I\right)=g\left(v_{i}\right)+I\left[h\left(v_{j}\right)-g\left(v_{i}\right)\right]$. This means that $f=g+q I=g+(h-g) I$. Now, we must prove that $g, q=h-g$ are classical linear transformations.
Let $x, y$ be any two elements of $V$, we have $x=x+0 I, y=y+0 I \in V(I)$. We have

$$
f(x+y)=f([x+0 I]+[y+0 I])=g(x+y)=g(x)+g(y) .
$$

For any $m \in F$, we have $m=m+0 I \in F(I)$, and $f([m+$ $0 I][x+0 I])=f(m x+0 I)=g(m x)=m g(x)$, and thus, $g$ is a linear transformation.

On the other hand, we have

$$
\begin{aligned}
x I, y I \in V(I), \text { and } f(x I+y I) & =f([x+y] I)=f(0+[x+y] I)=g(0)+I[(g+q)(x+y)-g(0)]=I[h(x+y)] \\
& =h(x) I+h(y) I, \text { thus } h(x+y)=h(x)+h(y), \\
f[m+0 I][0+x I] & =f(0+m x I)=g(0)+I[(g+q)(m x)-g(0)]=I[h(m x)] \\
& =m h(x) I, \text { so that } h(m x)=m h(x) .
\end{aligned}
$$

This implies that $g, h$ are two classical linear transformations; thus, $g, q$ are linear transformations, which implies that $f=g+q I$ is a full AH-linear transformation.

Remark 1. From Theorem 5 and Theorem 3, we get the following interesting result: every neutrosophic linear transformation $f: V(I) \longrightarrow W(I)$ can be represented by a unique neutrosophic matrix $M=A+B I$.

3.1. Further Applications. According to this work, we can use linear functions to study any problem that needs neutrosophic matrices. From this point of view, single-valued neutrosophic matrices used in [19] can be turned into algebraic linear functions.

\section{Conclusion}

In this paper, we have proved that every neutrosophic matrix can be represented uniquely by a neutrosophic linear vector space transformation. Also, we have showed that the linear property of any neutrosophic vector space function implies the AH-structure of this function.

This work opens a wide door to use neutrosophic vector spaces and matrices in classical representation theory of groups since it is well known that classical groups are represented by linear transformations from a vector space to itself. According to our results, we can find an important application of neutrosophic algebraic theory in the classical representation theory of groups. This application can be summarized by the following open question.

\section{Open Problem}

Determine the algebraic structure of all groups which can be represented by neutrosophic linear transformations from a neutrosophic vector space $V(I)$ to itself.

\section{Data Availability}

The data used to support the findings of this study are available from the corresponding author upon request.

\section{Disclosure}

This research has no external funding.

\section{Conflicts of Interest}

The author declares that there are no conflicts of interest.

\section{References}

[1] F. Smarandache, Introduction to Neutrosophic Statistics, Sitech \& Educ89, Columbus. OH, USA.

[2] F. Smarandache, "Neutrosophic set a generalization of the intuitionistic fuzzy sets," International Journal of Pure and Applied Mathematics, vol. 24, pp. 287-297, 2005.

[3] M. Abobala, "Classical homomorphisms between n-refined neutrosophic rings," International Journal of Neutrosophic Science, vol. 7, pp. 74-78, 2020.

[4] M. Abobala, "Semi homomorphisms and algebraic relations between strong refined neutrosophic modules and strong neutrosophic modules," Neutrosophic Sets and Systems, vol. 39, 2021.

[5] H. Sankari and M. Abobala, "Neutrosophic linear diophantine equations with two variables," Neutrosophic Sets and Systems, vol. $38,2020$.

[6] M. Abobala, "Foundations of neutrosophic number theory," Neutrosophic Sets and Systems, vol. 39, 2021.

[7] M. Abobala, "On some neutrosophic algebraic equations," Journal of New Theory, vol. 33, 2020.

[8] S. A. Edalatpanah, "Systems of neutrosophic linear equations," Neutrosophic Sets and Systems, vol. 33, pp. 92-104, 2020.

[9] T. Chalapathi and L. Madhavi, "Neutrosophic boolean rings," Neutrosophic Sets and Systems, vol. 33, pp. 57-66, 2020.

[10] M. Abdel-Basset, R. Mohamed, A. E. N. H. Zaied, A. Gamal, and F. Smarandache, "Solving the supply chain problem using 
the best-worst method based on a novel Plithogenic model," in Optimization Theory Based on Neutrosophic and Plithogenic Sets, pp. 1-19, Academic Press, Cambridge, CA, USA, 2020.

[11] W. B. V. Kandasamy and F. Smarandache, Neutrosophic Rings, Hexis, Phoenix, Arizona, 2006.

[12] M. Ali, F. Smarandache, M. Shabir, and L. Vladareanu, "Generalization of neutrosophic rings and neutrosophic fields," Neutrosophic Sets and Systems, vol. 5, pp. 9-14, 2014.

[13] F. Smarandache and M. Abobala, "n-Refined neutrosophic vector spaces," International Journal of Neutrosophic Science, vol. 7, pp. 47-54, 2020.

[14] H. Sankari and M. Abobala, "Solving three conjectures about neutrosophic quadruple vector spaces," Neutrosophic Sets and Systems, vol. 38, 2020.

[15] M. A. Ibrahim, A. A. A. Agboola, B. S. Badmus, and S. A. Akinleye, "On refined neutrosophic vector spaces I," International Journal of Neutrosophic Science, vol. 7, pp. 97109, 2020.

[16] A. A. A. Agboola and S. A. Akinleye, "Neutrosophic vector spaces," Neutrosophic Sets and Systems, vol. 4, pp. 9-17, 2014.

[17] M. Dhar, S. Broumi, and F. Smarandache, "A note on square neutrosophic fuzzy matrices," Neutrosophic Sets and Systems, vol. 3, pp. 37-41, 2014.

[18] H. Khaled, A. Younus, and A. Mohammad, "The rectangle neutrosophic fuzzy matrices," Faculty of Education Journal, vol. 15, 2019 Arabic version.

[19] P. K. Singh, "Single-valued neutrosophic context analysis at distinct multi-granulation," Computational and Applied Mathematics, June, vol. 38, p. 80, 2019, https://link.springer. com/article/10.1007/s40314-019-0842-4.

[20] M. Abobala, "A study of AH-substructures in n-refined neutrosophic vector spaces," International Journal of Neutrosophic Science, vol. 9, pp. 74-85, 2020.

[21] M. Abobala, "On some special elements in neutrosophic rings and refined neutrosophic rings," Journal of New Theory, vol. 33, 2020.

[22] F. Smarandache and M. Abobala, "n-Refined neutrosophic rings," International Journal of Neutrosophic Science, vol. 6, pp. 83-90, 2020.

[23] H. Sankari and M. Abobala, "AH-homomorphisms in neutrosophic rings and refined neutrosophic rings," Neutrosophic Sets and Systems, vol. 38, 2020.

[24] H. Sankari and M. Abobala, "n-Refined neutrosophic modules," Neutrosophic Sets and Systems, vol. 36, 2020. 